\title{
Auditoría financiera como herramienta de análisis de los estados financieros de la empresa Imgrumasa S.A. de la ciudad de Machala, periodo 2018
}

The financial audit as a tool for analyzing the financial statements of the company Imgrumasa S.A. of the city of Machala, during the period 2018.

Ronal Antonio Benavides Ortega. ${ }^{1}$, Cecilia Ivonne Narváez Zurita. ${ }^{2}$ Juan Carlos Erazo Álvarez. ${ }^{3}$ \& Yanice Licenia Ordoñez Parra. ${ }^{4}$

DOI: https://doi.org/10.33262/visionariodigital.v3i2.1.589

\begin{abstract}
.
The information generated by companies is very important for all those who integrate its, constituting a fundamental tool in the financial, administrative and operational fields, therefore, based on the Generally Accepted Auditing Standards, International Auditing Standards, Internal Control and the documentary review of several recognized authors in the area, this article shows the process of a financial audit applied in the company Imgrumasa SA of Machala city, during the period 2018, for this, they were employed

Procedures to examine and control the accounting information through the COSO I components, surveys and interviews that were addressed to the members of the Board of Directors, General Manager, Accountant and Commissioner of the entity. As a result of the audit, the deficient controls have been identified in different activities of the company that generate information for the preparation of the financial, as well as the lack of policies and procedures that frame the actions of the accountant.
\end{abstract}

\footnotetext{
1 Universidad Católica de Cuenca, Posgradista Maestría en Contabilidad y Auditoría, Cuenca, Ecuador, rabenavideso466@psg.ucacue.edu.ec

${ }^{2}$ Universidad Católica de Cuenca, Subdirección de Posgrado, Cuenca, Ecuador, inarvaez@ucacue.edu.ec

${ }^{3}$ Universidad Católica de Cuenca, Subdirección de Posgrado, Cuenca, Ecuador, jcerazo@ucacue.edu.ec

${ }^{4}$ Universidad Católica de Cuenca, Subdirección de Posgrado, Cuenca, Ecuador, jordonezp@ucacue.edu.ec
} 
Keywords: audit, financial, information, control, reliability.

\section{Resumen.}

La información que generan las empresas es de mucha importancia para todos aquellos que la integran, constituyéndose en una herramienta trascendental dentro del campo financiero, administrativo y operativo, por consiguiente, basados en las Normas de Auditoría Generalmente Aceptadas, Normas Internacionales de Auditoría, Modelos de Control Interno y la revisión documental de diversos autores reconocidos en el área, se muestra en el presente artículo el proceso de una auditoría financiera aplicado en la empresa Imgrumasa S.A. de la ciudad de Machala, en el periodo 2018, para ello, se emplearon procedimientos para examinar y controlar la información contable mediante los componente COSO I, encuestas y entrevistas que fueron dirigidas a los Miembros del Directorio, Gerente General, Contadora y Comisario de la entidad. Como resultado de la auditoría se identificaron los deficientes controles en las distintas actividades de la empresa que generan información para la elaboración de los estados financiero, así como, la carencia de políticas y procedimientos que enmarquen el accionar del responsable de la contabilidad.

Palabras claves: auditoría, financiera, información, control, fiabilidad

\section{Introducción.}

El mundo empresarial está lleno de un sin número de actividades enfocadas al crecimiento y desarrollo de las organizaciones e igual de las personas que conforman las mismas. Hoy en día crear una empresa no genera un grado de complejidad, mantenerse en el mercado es lo difícil por el nivel de competitividad que los diferentes sectores mantienen, con la existencia de la oferta y la demanda en base a la necesidad que los seres humanos requieren, generando movimientos económicos en relación a los ingresos y egresos.

Las dificultades que se presentan en las entidades deben ser solucionadas en un tiempo óptimo, para ello, deben utilizar de manera eficiente y eficaz sus recursos para la solución y alcance de las metas establecidas.

Todo ente contable sea público o privado, con o sin fines de lucro posee información financiera en relación a la variedad de transacciones que realizan, provocando entradas y salidas de dinero día a día. En este sentido, los estados de situación financiera tienen que ser claros, precisos y comparativos, en donde se refleje el correcto uso de los recursos, la transparencia, la fiabilidad y el efectivo funcionamiento de la actividad empresarial 
ocasionando un crecimiento transparente, niveles de confianza altos y viabilidad en la vida de la empresa.

El correcto uso de la información permite reconocer las anomalías que un ente pudiera tener y en el mejor de los casos contribuir con soluciones a la problemática, desde un área diferente como es la contable, indicando el punto específico o la variable defectuosa que estén generando las inconsistencias en la entidad, dando la pauta desde el departamento mencionado a la solución que esté requiriendo en su momento. Si los estados financieros no presentan la confiabilidad ante los máximos representantes, accionistas o dueños, generarán un ambiente de incertidumbre en la que se convertirán en un obstáculo para el buen y correcto funcionamiento en el alcance de los objetivos propuestos.

\section{Generalidades de la auditoría financiera}

En la actualidad es impensado asumir un mundo financiero sin procesos de auditoria a los estados financieros ejercidos por un profesional, ya que estos se constituyen en un soporte de confianza que el auditor da a los usuarios internos y externos. Por consiguiente, no se trata de un análisis frío de las cifras de una empresa, sino del análisis de las cifran contables que versan en los estados financieros y su incidencia en la determinación de la situación operativa, administrativa de producción o de servicios, aparados en normas internacionales de auditoría.

$\mathrm{Al}$ respecto, las normas de auditoría generalmente aceptadas (NAGAS) están vinculadas de forma cualitativa con los profesionales de la contabilidad, ocasionando mediante su excelente razonamiento, la redacción de un informe profesional en relación a las investigaciones realizadas por parte de los diferentes organismos de control competentes, tomando a la normativa vigente como documento de soporte para la sustentación de los pasos y aplicación de correctivos (Cardozo, 2016).

Las NAGAS según Paredes (2015) se dividen en normas generales o personales, normas de ejecución del trabajo, y las normas de preparación del informe, éstas resumen los procesos de auditoría desde el inicio hasta la presentación de los informes donde se demostrarán las evidencias encontradas en los estudios realizados. En la figura 1 se representan las normas. 
Figura 1. Clasificación de las normas de auditoría generalmente aceptadas.
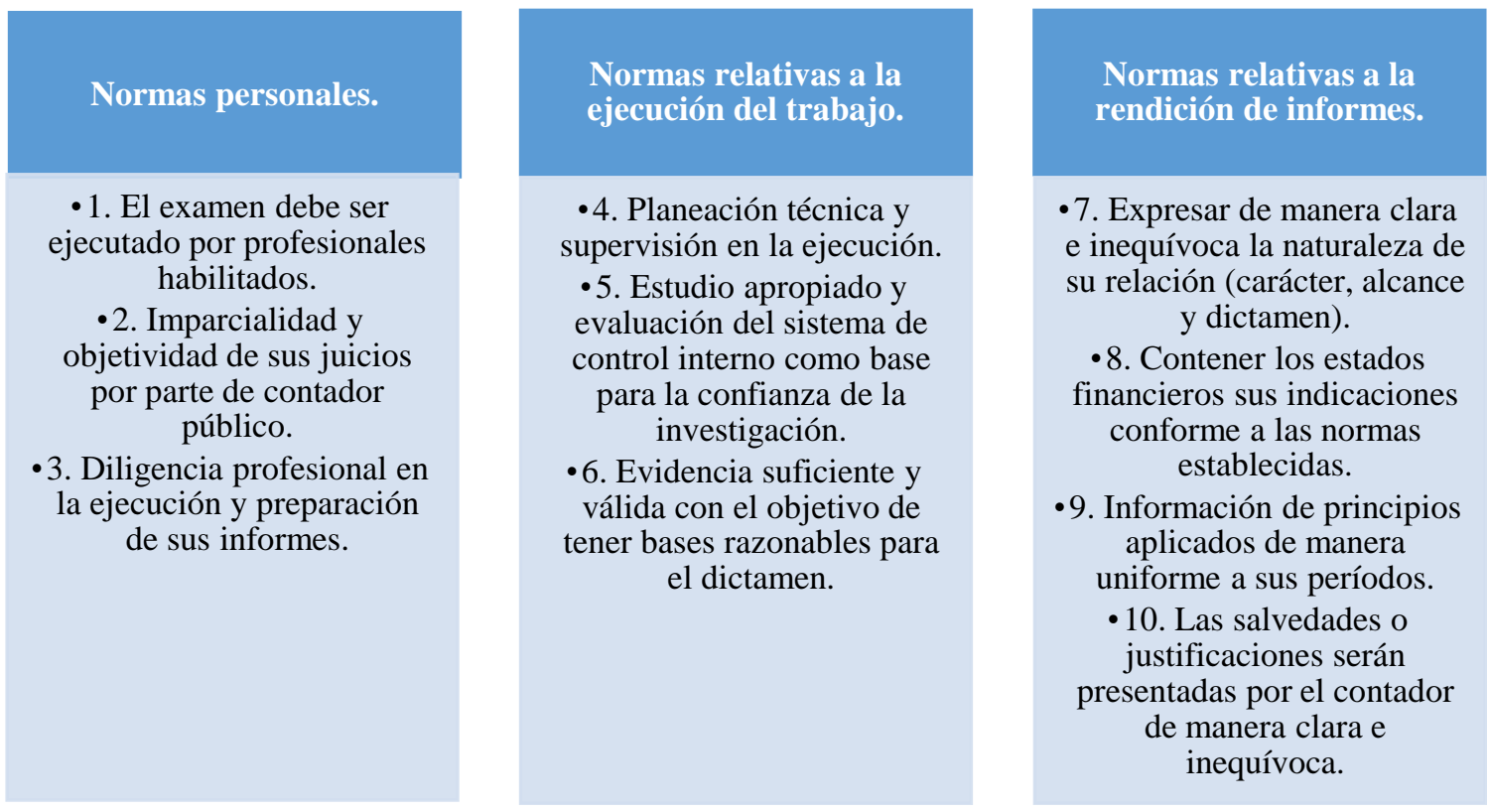

Fuente: Cardozo (2016)

Estupiñán (2013) señala que las normas internacionales de auditoría están comprendidas por diferentes destrezas o habilidades que, mediante la observación, identificación y sustentación de evidencias, permiten respaldar el informe en relación a los estados financieros auditados. Por su parte, Varela, Venini y Scarabino (2013) manifiestan que en el ámbito internacional la Federación Internacional de Contadores (IFAC) ha liderado la normativa en el campo de evolución de la auditoría, con el ingreso de empresas transnacionales o inversionistas de otros países, quienes están en la obligación de emitir normas de carácter internacional que permitan la sustentabilidad y transparencia en la emisión de un criterio profesional relacionado con la información obtenida, cabe señalar que cada país tiene sus propias normas y códigos de ética, sin embargo, la adopción de estándares internacionales garantiza una mayor comparabilidad en el entorno financiero, mayor confiabilidad en el esquema de normas y mayor promoción de inversión extranjera.

En este contexto, Muñoz (2002) define a la auditoría financiera como la revisión autónoma en relación a la variedad de movimientos que realizan las diferentes entidades, así como las funciones específicas, derivaciones y sistematizaciones de las organizaciones tratadas, realizadas por el profesional competente en la materia, con el propósito de emitir su criterio entorno a la información recopilada y el cumplimiento de las mismas de forma fiable y transparente, demostrando su eficiencia como profesional. 
Mientras que, Mantilla (2009) afirma que la auditoría financiera es una estructuración sistemática, basada en habilidades, métodos y técnicas por parte un profesional o un grupo inmerso en la materia a investigar, se enmarcada en los Estándares Internacionales de Auditoría (ISAs), los Estándares Internacionales de Control de Calidad (ISQCs), los Estándares Internacionales de Información Financiera (IFRS), los Estándares Internacionales de Contabilidad del Sector Público (IPSAS), y cualquier equivalente nacional e internacional.

Por su parte, Sánchez (2015) indica que, la auditoría financiera consiste en la verificación de los estados de situación financiera, en donde al auditor se le permite plasmar su juicio en base al examen realizado en el proceso de investigación en la entidad auditada, respetando los estándares internacionales de información financiera, mostrando la transparencia, legibilidad y determinando la validez y confiabilidad de la información. El autor hace énfasis en que la única persona que tiene la potestad de dictar su posición como profesional sobre los estados financieros es el auditor. Sin embargo, no se puede asumir que la profesión del auditor se enmarca únicamente en la opinión de los estados financieros, ya que este profesional puede ejercer amplias funciones de asesoría y emitir diferentes de opiniones dependiendo del tipo de auditoría que realice.

Tabla 1 Clasificación de la auditoría

\begin{tabular}{lll}
\hline \multicolumn{1}{c}{ Clasificación } & \multicolumn{2}{c}{ Tipos de auditoría } \\
\hline 1. La afiliación o el sector al que & - Auditoría estatal & - De obra pública \\
pertenece el auditor & - Estatal general & - De resultado de programas \\
& - Estatal fiscal & - Presupuestaria \\
2. La relación que media entre el & - Independiente & \\
cliente y el auditor respecto a su & - Auditoría externa & - Auditoría interna \\
independencia mental & & \\
& - Auditoría financiera & - Social \\
& - De control interno & - Ambiental \\
& - De cumplimiento & - De recursos humanos \\
& - De gestión & - De calidad \\
3. El objeto de estudio & - Tributaria privada & - De la información \\
& - De contratos & - De sistemas informáticos \\
& - Laboral & - Forense \\
& - Administrativa & - De riesgos \\
& - Operacional & \\
4. La periodicidad con que se practique & - Auditorías periódicas & - Continuas \\
\end{tabular}

Fuente: Montilla y Herrera (2006) 
Tapia, Guevara, Castillo, Rojas y Salomón (2016) clasifican a la auditoría en base a las diferentes funciones que se pueden realizar entre las múltiples entidades existentes, afirman que todo puede ser auditable, considerando que las responsabilidades no deben caer sobre el contador en su totalidad, existen varias disciplinas entorno a la empresa donde la rama de la contabilidad no domina algunos métodos y se requiere de varias profesiones, su clasificación es muy variada como: la auditoría financiera, interna, externa, administrativa, integral, gubernamental, informática y todo aquello que ejerza funciones en un ente.

La auditoría financiera permite a las entidades que manejan flujos de efectivo transparentar los ingresos y gastos que repercuten en su producción o consumo, de tal forma que, a través de sus estados financieros identifica el correcto uso de los recursos. Para llegar al cumplimiento del proceso a auditar, es indispensable la aplicación de etapas la investigación, permitiendo la utilización de métodos y técnicas que agiliten el objetivo a cumplir. (Morales, 2013)

Figura 2. Esquema de la auditoría financiera

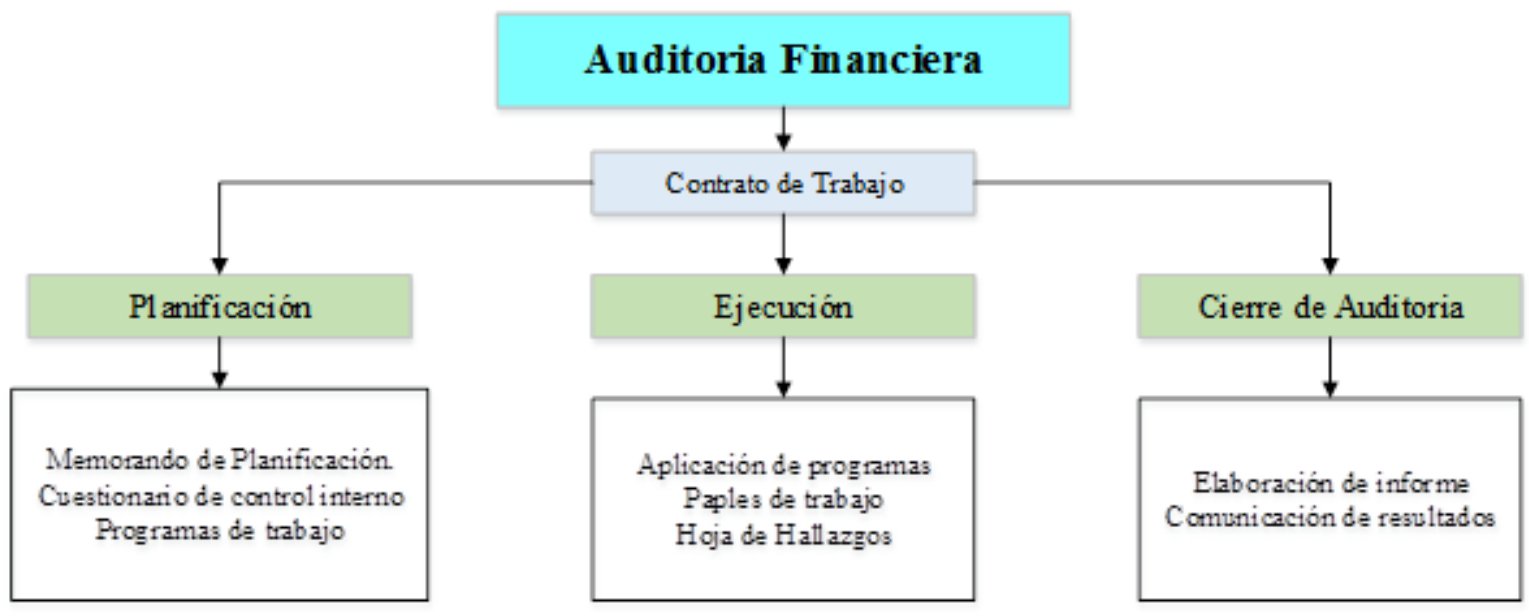

La etapa de planificación de la auditoría según Sánchez (2015) profundiza en los aspectos más característicos de los estados de situación financiera, ya que permite investigar la estructura organizacional de la empresa, generar un alto grado de confianza, tomar como referencia las evidencias adquiridas en el transcurso de su proceso de investigación efectuado y procurar la obtención de datos relevantes para las futuras conclusiones. Tapia, Guevara, Castillo, Rojas y Salomón (2016), aseguran que la etapa de planificación debe culminar en el periodo acordado con los representantes del ente auditado o en el tiempo establecido por los organismos de control.

La etapa de ejecución en auditoría tiene como objetivo principal la formación de un criterio sobre el raciocinio de la investigación de los estados financieros, mediante la realización de 
diferentes tipos de pruebas y análisis para determinar su razonabilidad. Al respecto, Tapia, Guevara, Castillo, Rojas y Salomón (2016) manifiestan que es fundamental la confianza depositada en el control interno, ya que, con ello, la revisión hecha en esta etapa, permitirá al auditor agilitar de forma eficiente y oportuna el dictamen a presentar en la etapa final.

Para el cierre de la auditoría, Sánchez (2015) se enfoca de manera significativa en la actualización de las recomendaciones emitidas en los procesos anteriores, así como la revisión realizada en los estados financieros, la documentación obtenida y todos aquellos factores que han influido en su proceso de investigación, permitiendo al auditor proyectar su criterio profesional de forma consolidada, facilitándole a la entidad conocer sus falencias y virtudes entorno al periodo analizado.

Por lo expuesto, se considera que las etapas de la auditoría son muy importantes, por lo que parten de un proceso de investigación para la revisión de los estados financieros en el que se van evidenciando los diferentes movimientos que se han suscitado en la entidad durante un periodo establecido, cada etapa depende de la anterior por lo que permite avanzar de manera fiable hasta la presentación del dictamen final, reflejando si la situación mostrada por el ente tiene veracidad, contribuyendo en el transcurso del estudio con la redacción de las recomendaciones y sugerencias para el mejoramiento institucional, así mismo, ayuda a la empresa a reconocer las virtudes y defectos que posee, facilitando al auditor tener una mayor claridad y seguridad para plasmar su criterio profesional.

\section{Control interno}

El control interno es el plan organizacional donde se amparan los altos directivos o miembros de una empresa, acerca de sus bienes e información significativa; afirmando la fiabilidad y el razonamiento en los estados financieros y presupuestarios; lo cual promueve la eficiencia en las diferentes funciones realizadas de forma cronológica y sistematizada, esto conlleva a cumplir los objetivos trazados por la entidad mediante la seguridad razonable adquirida en el transcurso del proceso de investigación hecha por el profesional de campo enfocada en su cumplimiento y en el crecimiento empresarial (Plasencia, 2010). Con el fin de proteger los bienes y cumplir los objetivos planteados para la empresa, el control interno es una herramienta de gran importancia para la entidad, por lo que permite ayudar y controlar el funcionamiento correcto del ente, incorporando técnicas, procedimientos en su proceso y la normativa vigente para el respaldo de su accionar en las distintas funciones a efectivizar.

El control interno tiene como objetivo principal salvaguardar los bienes y el cumplimiento de las metas impuestas por la organización, en un periodo establecido. Al respecto, Infante et al. (2017) consideran que, el examen de control interno se enfoca en realizar una 
evaluación eficiente y confiable en la entidad a estudiar, para ello, el profesional competente debe poseer una despejada visión acerca del comportamiento de las diferentes etapas del control interno, así como la evidencia necesaria para poder respaldar los criterios profesionales emitidos.

La evaluación del control interno en la empresa, inicia con la elaboración de un cuestionario de preguntas donde se detalla los componentes del modelo de control interno a ser aplicado, intentando identificar las fortalezas y debilidades que posee el ente auditado en las actividades que realiza, a través de ello, se descubre los riesgos de su entorno y aplica el tratamiento correspondiente, aportando en la ejecución de los correctivos para la clarificación y transparencia de la situación de la entidad.

\section{Programas de trabajo}

Venegas (2007) afirma que los programas de trabajo son un conjunto de actividades que se aplican en una auditoría, donde el auditor es el encargado de elaborarlos para su debido proceso, los mismos, permiten al profesional de campo llevar un control cronológico y sistematizado de su cumplimiento.

En el programa de auditoría se enmarcan las actividades a realizar durante el proceso, el mismo se encuentra detallado en la etapa de planificación, entra en funcionamiento en la etapa de ejecución donde a través de los papeles de trabajo se puede evidenciar los distintos hallazgos encontrados, permitiendo obtener evidencias que contribuyen con la clarificación de la información financiera y sustentabilidad en el informe final.

\section{La información contable en las empresas}

Para Arias, Portilla y Agredo (2007), lo más importante en la información contable que generan las empresas es que su fuente sea de confianza en relación a las cifras mostradas en sus estados financieros, por consiguiente, se deben realizar controles durante el proceso contable, así como poseer políticas, reglamentos o leyes que contribuyan a la elaboración y presentación de los estados financieros.

En este sentido, Carrasco y Pallerola (2014) sustentan que los estados financieros presentados bajo las Normas Internacionales de Información Financiera (NIIF), tienen dos objetivos que contemplan el desempeño empresarial y el desempeño administrativo, abordando el primero, éste hace referencia a la razonabilidad de la información, la rentabilidad obtenida y los flujos de efectivo que se presentan a cada uno de los usuarios de la información, en tanto que, el desempeño administrativo, se refiere a la evaluación de la gestión que se realiza en la parte administrativa, logrando medir la efectividad de la administración en el correcto uso y control 
de los recursos. Los estados financieros tienen como objetivo principal, el suministro de información contable, en la que se visualiza la labor de la parte administrativa en la empresa, por ende, considerando su nivel de importancia, resulta necesarios validar dicha información a través de la auditoría financiera, de tal manera que los usuarios de la información cuenten con la certeza de que la misma es confiable, veraz y razonable.

\section{Metodología.}

La presente investigación se desarrolló bajo un enfoque mixto, ya que, se sustentó en aspectos cuantitativos y cualitativos para el análisis del objeto de estudio. En la primera fase de la investigación mediante aproximaciones cualitativas se analizaron la estructura de las variables de estudio, mientras que, en la fase de diagnóstico y desarrollo de la propuesta se integraron sistémicamente los métodos cuali - cuantitativos a fin de obtener evidencia numérica, verbal, textual y visual sobre la información contable de la empresa.

Considerando las características de la investigación, ésta se desarrolló bajo dos alcances, en esencia se enmarcó en el alcance explicativo, con contenido de elementos descriptivos. El alcance descriptivo permitió especificar las propiedades y características de la auditoría financiera e información contable sometidas a análisis. Por su parte, el alcance explicativo contribuyó con la identificación de las causas que generaron eventos no favorables sobre la información contable elaborada por la entidad. Es decir, éste alcance se enfocó en explicar la ocurrencia del fenómeno en estudio y su relación con la auditoría financiera.

Como procedimiento de investigación del presente informe se utilizó la técnica de la encuesta, ya que, permitió obtener y elaborar datos de forma rápida y eficaz mediante la utilización de dos cuestionarios. El objetivo que persiguieron estos instrumentos fue la traducción de variables empíricas sobre las que se obtuvo información en base a preguntas concretas que generaron información valida susceptible de hacer cuantificada. Los cuestionarios se estructuraron en base a preguntas cerradas y de selección múltiple con un abanico de respuestas. Cabe mencionar que, la guía de observación fue utilizada para recopilara aquella información que sucedía durante el transcurso de la estadía en la empresa.

\section{Resultados.}

Luego de haber aplicado los instrumentos de investigación se pudo obtener la información necesaria de los estados financieros de la empresa Imgrumasa S.A. de la ciudad de Machala, que demuestran la situación financiera actual por la que está atravesando, en los siguientes enunciados se plasman los resultados obtenidos: 


\section{Estado de situación financiera}

La evaluación realizada sobre el estado de situación financiera permitió recopilar información acerca de las cuentas de activo, pasivo y patrimonio, donde se puedo identificar:

$>$ Existencia de cuentas incobrables ante la carencia de políticas de cobro.

$>$ Realización de inventarios de mercaderías parciales sin supervisión.

$>$ Inexistencia de comprobación de las depreciaciones realizadas.

$>$ Carencia de controles en el endeudamiento de la empresa.

$>$ Incumplimiento en el pago a proveedores.

\section{Estado de resultado integral}

En la investigación realizada al estado de resultados, se logró obtener información acerca de las cuentas de ingresos y egresos de la entidad donde logró identificar:

Falta de comprobación entre el rubro de ingresos generados y el efectivo en caja.

$>$ Inconsistencia en la distribución y detalle en la cuenta otros gastos.

$>$ Cumplimiento de ventas presupuestadas.

$>\quad$ Bajo índice de liquidez.

\section{Estado de cambios en el patrimonio}

Con la información obtenida en la investigación al estado de patrimonio, se logró evidenciar:

> Inconsistencias en la aprobación de los porcentajes de reservas ante la máxima autoridad de la empresa.

$>$ Apertura de cuentas en el patrimonio sin previa autorización.

\section{Estado de flujo de efectivo}

$>$ Carencia de controles en los flujos de efectivo de la empresa.

$>$ Índices bajos de confianza ante la escasez de controles.

\section{Notas aclaratorias}

Inexistencia de supervisión a las notas aclaratorias. 
Con los resultados obtenidos, se evidencia la necesidad de realizar un proceso de auditoría a los estados financieros dentro del periodo 2018, con la finalidad de obtener razonabilidad y transparencia de la información, lo cual ayudará a la administración a tomar decisiones efectivas para el fiel cumplimiento de sus objetivos institucionales.

El proceso de auditoría realizado comprendió tres fases que son: la planificación, ejecución y cierre de auditoría.

Tabla 2. Esquema de la auditoría financiera a Imgrumasa S.A.

\begin{tabular}{llll}
\hline Fases & Actividad 1 & Actividad 2 & Actividad 3 \\
\hline I Planificación & $\begin{array}{l}\text { Memorando de } \\
\text { planificación }\end{array}$ & $\begin{array}{l}\text { Cuestionario de control } \\
\text { interno }\end{array}$ & $\begin{array}{l}\text { Programa de } \\
\text { trabajo }\end{array}$ \\
II Ejecución & $\begin{array}{l}\text { Aplicación de } \\
\text { programas }\end{array}$ & Papeles de trabajo & Hallazgo \\
$\begin{array}{l}\text { III Cierre de } \\
\text { auditoría }\end{array}$ & Elaboración de informe & $\begin{array}{l}\text { Comunicación de } \\
\text { resultados }\end{array}$ & \\
\hline
\end{tabular}

Dentro del proceso de auditoría financiera, se inició con la etapa de planificación, en esta etapa se estructuraron los componentes de los estados financieros a ser examinados, a continuación, se detallan los grupos considerados para el estudio.

IMGRUMASA S.A.

Desde 01 de enero de 2018 hasta 31 de diciembre de 2018

Estado De Situación Financiera

$\begin{array}{ll}\text { DETALLE } & \text { SALDO } \\ \text { Activo } & 304258,28 \\ \text { Pasivo } & 274431,58 \\ \text { Patrimonio } & 29826,70 \\ \text { Ingreso } & 592386,92 \\ \text { Egreso } & 585940,22\end{array}$

En la tabla 3, se evidencian los servidores relacionados durante el periodo de administración 2018, detallando los apellidos, nombres y cargos correspondientes. 
Tabla 3. Servidores relacionados

\begin{tabular}{llcc}
\hline \multicolumn{1}{c}{ Apellidos } & \multicolumn{1}{c}{ Nombres } & Cargo & Periodo de actuación \\
\hline Loayza Apolo & Roberth Patricio & Presidente & $01 / 01 / 2018-31 / 12 / 2018$ \\
Valarezo Loayza & Richard Eduardo & Gerente & $01 / 01 / 2018-31 / 12 / 2018$ \\
Martínez Añazco & Richard Gonzalo & Comisario & $01 / 01 / 2018-31 / 12 / 2018$ \\
Ochoa Jaramillo & Lourdes Paulina & Contadora & $01 / 01 / 2018-31 / 12 / 2018$ \\
\hline
\end{tabular}

\section{Componentes a ser examinados}

$\checkmark$ Balance General

$\checkmark$ Estado de Resultado

$\checkmark$ Estado de Cambios en el Patrimonio

$\checkmark$ Estado de Flujo de Efectivo

$\checkmark$ Notas Aclaratorias

Los puntos de interés identificados por componente, para su análisis en el proceso de auditoría son los siguientes:

$\checkmark$ Control y registro de las cuentas por cobrar que no han sido recaudadas durante más de dos años.

$\checkmark$ Cálculo, registro y pago de remuneraciones y demás beneficios que poseen los trabajadores en relación a la prestación de servicios.

$\checkmark$ Cálculo registro y pago a proveedores con mayor volumen de compras en el mes.

En la tabla 4 se detalla el programa de actividades correspondiente a la auditoría financiera en proceso, iniciando la acción de control el 01 de abril de 2019

Tabla 4. Días programados y fecha de intervención

\begin{tabular}{lllll}
\hline & Días Calendario & Desde & Desde \\
\hline I & $\begin{array}{l}\text { Planificación y programación } \\
\text { II Programado 30\% }\end{array}$ & 12 & $1 / 4 / 2019$ & $12 / 4 / 2019$ \\
$\begin{array}{l}\text { Ejecución } \\
\text { Programado 50\% }\end{array}$ & 20 & $13 / 4 / 2019$ & $2 / 5 / 2019$ \\
III & $\begin{array}{l}\text { Cierre de Auditoría } \\
\text { Programado 20\% }\end{array}$ & 7 & $3 / 5 / 2019$ & $9 / 5 / 2019$ \\
& 1 & $10 / 5 / 2019$ & \\
\hline Comunicación de Resultado & Total, Días & 40 & & \\
\hline
\end{tabular}


En la tabla 5 se detalla la distribución del tiempo en que se ejecutará la auditoría financiera cumpliendo cada una de sus fases:

Tabla 5. Distribución de trabajo

\begin{tabular}{lllll}
\hline \multirow{2}{*}{ Responsable } & \multicolumn{1}{c}{ Actividad } & \multicolumn{2}{c}{ Días } & \multicolumn{2}{c}{ Días programados } \\
& & calendario & Desde & Desde \\
\hline & Planificación & 12 & $1 / 4 / 2019$ & $12 / 4 / 2019$ \\
& Análisis de estados financieros. & & $13 / 4 / 2019$ & $02 / 5 / 2019$ \\
\multirow{2}{*}{$\begin{array}{l}\text { Ronal Antonio } \\
\text { Benavides }\end{array}$} & Elaboración de comentarios, conclusiones & & & \\
Ortega & y recomendaciones sobre los hallazgos & 20 & & \\
& determinados en el análisis. & & & \\
& Elaboración de informe & 7 & $3 / 5 / 2019$ & $9 / 5 / 2019$ \\
& Comunicación de resultado & 1 & $10 / 5 / 2019$ & $10 / 5 / 2019$ \\
& Total & 40 & & \\
\hline
\end{tabular}

Para la evaluación del riesgo en el proceso de auditoría financiera, se consideró las cuentas de grupo para su análisis, demostrando que los pasivos de la empresa no poseen los controles indicados para obtener un nivel de confianza moderado. En la tabla 7 se detalla el análisis de los componentes:

Tabla 6. Matriz de evaluación y calificación de los riesgos de auditoría financiera

\begin{tabular}{|c|c|c|c|c|c|}
\hline \multirow{2}{*}{ Componente } & \multicolumn{2}{|c|}{ Riesgo y su fundamento } & \multirow[t]{2}{*}{$\begin{array}{l}\text { Controles } \\
\text { claves }\end{array}$} & \multicolumn{2}{|c|}{ Enfoque de auditoria } \\
\hline & $\begin{array}{l}\text { Inherente } \\
\text { Moderado }\end{array}$ & $\begin{array}{l}\text { Control } \\
\text { Alto }\end{array}$ & & $\begin{array}{c}\text { Pruebas de } \\
\text { cumplimiento }\end{array}$ & Pruebas sustantivas \\
\hline ingresos & $\begin{array}{l}\text { Evidenciar } \\
\text { registro por } \\
\text { otros ingresos }\end{array}$ & $\begin{array}{l}\text { Falta de } \\
\text { custodia } \\
\text { por ingreso } \\
\text { generados } \\
\text { y } \\
\text { depositados } \\
\text { en caja }\end{array}$ & $\begin{array}{l}\text { Evidenciar } \\
\text { y controlar } \\
\text { los } \\
\text { ingresos } \\
\text { generados }\end{array}$ & $\begin{array}{l}\text { Verificar la existencia } \\
\text { de una adecuada } \\
\text { segregación de las } \\
\text { funciones } \\
\text { determinación, } \\
\text { recaudación de los } \\
\text { ingresos, su correcta y } \\
\text { oportuna } \\
\text { contabilización. }\end{array}$ & $\begin{array}{lr}\text { Verificar en los } \\
\text { comprobantes } \\
\text { ingresos, de } \\
\text { corrección de los } \\
\text { cálculos aritméticos, } \\
\text { secuencia numérica } \\
\text { progresiva, firmas de } \\
\text { responsabilidad }\end{array}$ \\
\hline
\end{tabular}


Pérdida de valores en ventas de efectivo

Incumplimiento

en la

facturación
Comprobar la Verificar que hayan utilización de reportes sido registrados todos pre numerado e impreso los ingresos sobre cobranzas y correspondientes al rentas recibidas como periodo bajo sustento de su auditoría. apropiado registro.

Verificar la secuencia numérica de las facturas, notas de débito y crédito del periodo de la muestra.

Verificar las facturas de la guía de remisión firmadas por los clientes

Tabla 7. Análisis del riesgo

\begin{tabular}{cccccc}
\hline No & Componentes & \multicolumn{2}{c}{ Nivel de confianza } & \multicolumn{2}{c}{ Riesgo de control } \\
\hline 1 & Activo & 74,40 & Moderado & 25,60 & Moderado \\
2 & Pasivo & 50,00 & Bajo & 50,00 & Alto \\
3 & Patrimonio & 66,67 & Moderado & 33,33 & Moderado \\
4 & Ingresos & 83,33 & Alto & 16,67 & Bajo \\
5 & Egresos & 83,33 & Alto & 16,67 & Bajo \\
\hline
\end{tabular}

Una vez cumplida con la etapa de planificación y analizado el nivel de riesgos de las cuentas de grupo, se procedió al desarrollo correspondiente de las actividades diseñadas, los hallazgos encontrados permitieron reflejar las falencias a través de su hoja comprobatoria, donde se logró evidenciar dos errores relacionados con el registro contable de los. A continuación, se presenta uno de los papeles de trabajos aplicados en este proceso.

Tabla 8. Estado de resultado integral

\begin{tabular}{llrrrr}
\hline Índice & \multicolumn{1}{c}{$\begin{array}{c}\text { Saldo } \\
\text { Nombre de las cuentas }\end{array}$} & $\begin{array}{c}\text { 2017-12- } \\
\mathbf{3 1}\end{array}$ & $\begin{array}{c}\text { Saldo } \\
\mathbf{2 0 1 8 - 1 2 - 3 1}\end{array}$ & $\begin{array}{c}\text { Ajustes y } \\
\text { reclasificaciones } \\
\text { Debe }\end{array}$ & $\begin{array}{c}\text { Saldo 2019- } \\
\text { 12-31 según } \\
\text { auditoría }\end{array}$ \\
$\begin{array}{l}\text { Ingresos de actividades } \\
\text { ordinarias }\end{array}$ & 546347,63 & 591283,54 & & 591283,54 \\
$4.2 \quad$ Ingresos no operacionales & 13,21 & 200,35 & & 200,35
\end{tabular}




\section{$\sqrt{ }$ Comprobado \\ $\sum$ Totalizado \\ S Documentación sustentadora}

Conclusión:

Los ingresos presentan en forma razonable los recursos de la empresa

En la tabla 9, se puede evidenciar a través de la condición, criterio, causa y efecto, las características del hallazgo:

Tabla 9. Hoja de Hallazgos

\section{Ing. Ronal Antonio Benavides Ortega Ing. Ronal Antonio Benavides Ortega Imgrumasa S.A. Imgrumasa S.A.}

Hoja de hallazgo 1 Hoja de hallazgo 2

\begin{tabular}{ll}
\hline Período: & $\begin{array}{l}\text { del } 01 \text { de enero de } 2018 \text { al } 31 \mathrm{de} \\
\text { diciembre de } 2018\end{array}$ \\
Componente & Estados Financieros \\
Ref. P/T: & PT EFEF \\
Titulo: & Elaboración de estados financieros \\
& \\
& $\begin{array}{l}\text { La empresa IMGRUMASA S.A. realizó } \\
\text { estados financieros de los cuales no } \\
\text { posee la entidad un manual de } \\
\text { procedimientos y políticas contables. }\end{array}$
\end{tabular}

del 01 de enero de 2018 al 31 de diciembre de 2018

Estados Financieros

PT EFCI

Justificación de la cuenta otros ingresos

La empresa IMGRUMASA S.A. realizó ingresos no operacionales en relación a la actividad que ejerce, donde no existen respaldos comprobatorios y controles que den veracidad al rubro establecido y justifique el $100 \%$ en la entrega del efectivo.

Incumple con lo establecido en la NIC

Criterio: $\quad 8$, Políticas contables, cambios en las estimaciones contables y errores

No presenta controles internos en la actividad por lo que la aplicación del código de ética como profesional es poco relevante.

La contadora de la empresa realizó los

Se realizó el ingreso no operacional sin la estados financieros bajo políticas de su Causa: experiencia como profesional, al no poseer internamente un manual de procedimientos y políticas contables. documentación que propicie veracidad y responsabilidad en la totalidad de la venta de aceite quemado y filtros usados, donde no existe una valoración eficiente para su venta. 
Los estados financieros generan un nivel de poca confianza entre sus accionistas al no estar amparados durante su

Efecto: elaboración bajo un manual de procedimientos y políticas contables en las que les permita controlar el proceso contable.

Diseñar un manual de procedimientos y Conclusión políticas contables para el aumento de la fiabilidad de la información contable

Desconfianza en los valores entregados a la empresa.

Incumplimiento en la emisión de sustentos de ventas.

Una vez cumplida la planificación y ejecutada la auditoría financiera, se analizó las evidencias recopiladas para la elaboración del respectivo informe de auditoría, donde se enfoca en los aspectos que generan las debilidades en la empresa, permitiendo a que Imgrumasa S.A., demuestre de una forma trasparente la confianza que requieren los estados financieros. Algunas deficiencias se pueden corroborar mediante controles en las distintas áreas y plasmando políticas que ayuden a los estados financieros en el proceso contable y su presentación.

Concluida la auditoría financiera y elaborado su informe, se procedió a comunicar a la empresa las conclusiones con criterio profesional, permitiendo a través del proceso realizado contribuir con la transparencia, razonabilidad y confiabilidad de la información financiera del ente, quedando el informe de la siguiente forma:

\section{Informe de auditoría sobre los estados financieros de la empresa Imgrumasa S.A.}

\section{(Ejercicio finalizado al 31 de diciembre de 2018)}

La presente auditoría financiera realizada en la empresa Imgrumasa S.A. de la ciudad de Machala tiene fines académicos, en tal razón pretende dar solución a problemas contables identificados.

\section{Objetivos de la auditoria}

$\checkmark$ Emitir una opinión independiente respecto a si los estados financieros de la empresa Imgrumasa S.A. presentan razonablemente en todos los aspectos significativos la situación patrimonial, financiera, resultados de sus operaciones, y flujo de efectivo.

$\checkmark$ Determinar si los controles internos establecidos, guardan relación con la presentación de los estados financieros. 


\section{Alcance}

El alcance de la auditoría financiera cubrió las operaciones realizadas por la empresa Imgrumasa S.A., en el período comprendido entre el 01 de enero de 2018 hasta 31 de diciembre de 2018. Para ello, se tomó en consideración las Normas Internacionales de Auditoría y las Normas de Auditoría Generalmente Aceptadas, las cuales fueron incluidas en los procedimientos mínimos de auditoría.

\section{Base legal}

IMGRUMASA S.A esta constituida desde el 31 d enero de 2003, la empresa se dedica a la venta e importación de repuestos para buses y camiones, cuenta con 169 accionistas y 20040 acciones, se encuentra identificada en el campo mercantil como una compañía anónima, por lo que su organismo de control es la Superintendencia de Compañías.

Figura 3. Estructura orgánica

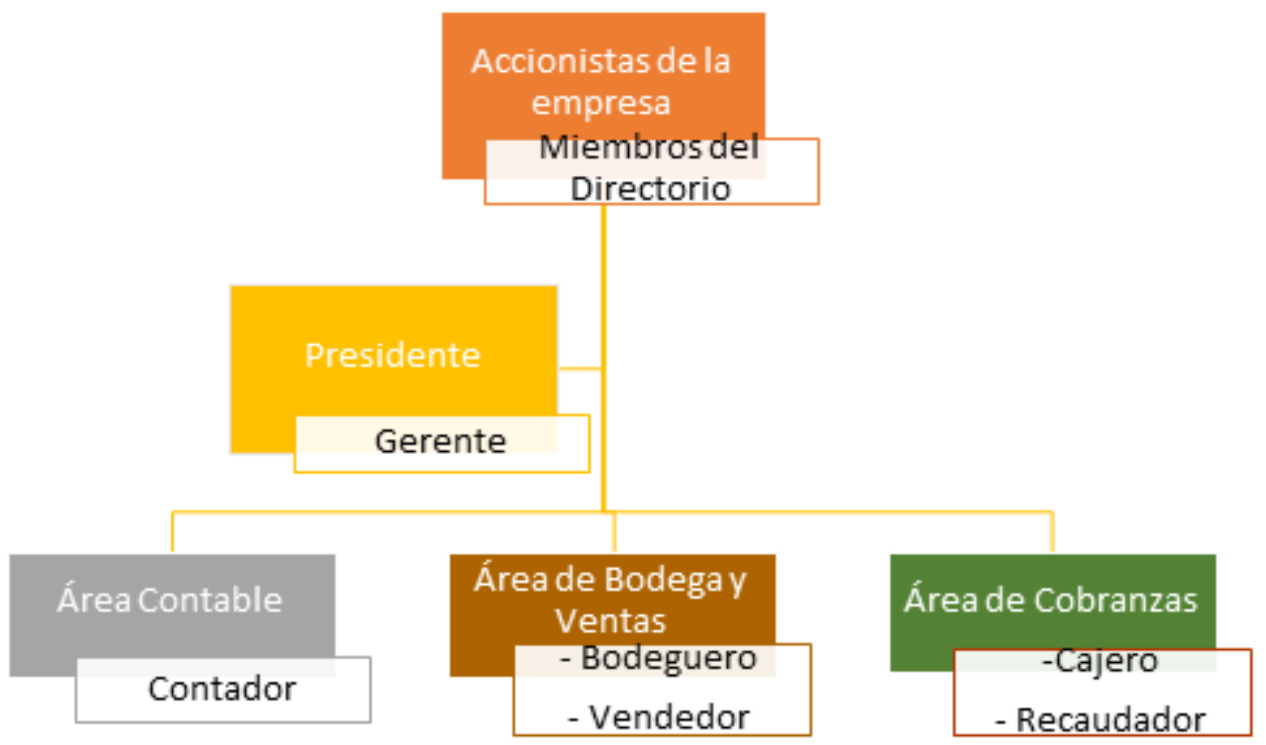

\section{Objetivo general institucional}

Brindar asistencia técnica en la venta de repuestos, partes y pieza; seguir siendo un apoyo para los socios de la cooperativa Ciudad de Machala y de los transportistas de El Oro en general. 


\section{Dictamen}

He auditado los estados financieros de la empresa Imgrumasa S.A. que comprende el estado de situación financiera, el estado de resultado integral, el estado de cambios en el patrimonio, el estado de flujo de efectivo y las notas aclaratorias del periodo contable 2018.

\section{Responsabilidad de la administración en relación con los estados financieros}

La transparencia y razonabilidad en la elaboración y presentación de los estados financieros de acuerdo a las normas internacionales de información financiera es de responsabilidad de la administración, así como la aplicación de un control interno en las actividades que se ejecutan diariamente a efectos de no permitir una desviación material que propicie fraude o error durante su proceso de preparación.

\section{Responsabilidad del auditor}

Mi responsabilidad es emitir un criterio acerca de los estados financieros a través de una auditoría. La auditoría se ha llevado a cabo de acuerdo a las normas internacionales de auditoría, donde a través de su exigencia, se permite cumplir con actos éticos para la correspondiente planificación y ejecución con el propósito de obtener la seguridad y razonabilidad para la emisión de un criterio profesional.

En el desarrollo de la auditoría, se aplicaron procedimientos para la obtención de evidencia, seleccionándola desde la suficiencia, competencia, importancia y pertinencia por medio de las distintas técnicas aplicadas a los estados financieros. Para la evaluación del riesgo, se consideró el control interno implementado en la entidad, donde se aplicó los procedimientos idóneos acorde a la actividad de la empresa.

\section{Opinión}

En mi opinión, los estados financieros presentan la razonabilidad necesaria acorde al periodo 2018 en la empresa Imgrumasa S.A. encontrando como resultado:

1. Se realizó la presentación de los estados financieros comprendida en el periodo contable 2018, donde se pudo detectar que las diversas actividades que intervienen en un proceso contable, son elaboradas de forma empírica e incumple la norma internacional de contabilidad 8 en base a las distintas políticas contables que debe poseer la empresa, registrada en un manual para el cumplimiento eficiente en el proceso contable en relación a un protocolo institucional, generando inseguridad en los resultados obtenidos. 
2. Se ejecutaron ingresos no operacionales en relación a la actividad que ejerce, donde no existen respaldos comprobatorios y controles que den veracidad al rubro establecido y justifique el $100 \%$ en la entrega del efectivo. Aquí se pudo evidenciar la ausencia de procedimientos de control interno y un código de ética que rija el comportamiento de los trabajadores, por lo que, al no existir controles durante el desarrollo de la actividad, se genera desconfianza en los valores entregados y debilidades en el soporte de las transacciones generadas.

NN.

10 de mayo de 2019

Machala - Ecuador

\section{Conclusiones.}

- La auditoría financiera tiene por finalidad dictaminar la razonabilidad de los estados financieros, a partir de ello, se genera información financiera confiable que sirve de soporte para la toma de decisiones.

- La contabilidad y la auditoría se encuentran en permanente evolución, lo que exige que los auditores participen en procesos de capacitación continua a fin de que acumulen conocimientos que permitan que las actividades a ellos encomendados sean cumplidas eficientemente.

- La auditoría financiera aplicada a la empresa Imgrumasa S.A. cumplió con su objetivo, permitiendo clarificar y entender el tratamiento que se le daba a las distintas cuentas contables y el cumplimiento de las disposiciones de las normas vigentes relacionadas con los procesos tributarios y laborales.

- Finalmente, el desarrollo de la presente investigación genera una herramienta ideal para el directorio de la compañía, ya que se puede apoyar en el mismo para fortalecer los mecanismos de control interno y generar información contable oportuna y veraz. 


\section{Referencias bibliográficas.}

Arias, L., Portilla, L., \& Agredo, L. (2007). Información contable en el análisis de los estados financieros. Red de Revistas Científicas de América Latina, el Caribe, España y Portugal., XIII, 311-314. Obtenido de https://www.redalyc.org/pdf/849/84903753.pdf

Cardozo, H. (2016). Auditoría del sector solidario. Bogotá: Ecoe Ediciones Ltda.

Carrasco, C., \& Pallerola, J. (2014). Gestión financiera. Bogotá: Rama.

De la Peña, A. (2011). Auditoría un enfoque práctico. Madrid: Paraninfo S.A.

Estupiñán, R. (2013). Papeles de trabajo en la auditoría financiera: con base en la NIA. Bogotá: Ecoe Ediciones.

Infante, A., García, R., Galindo, V., Baza, F., Barragán, D., Ruíz, F., \& León, M. (2017). Normas de auditoría para atestiguar, revisión y otros servicios relacionados. México: México.

Mantilla, S. (2009). Auditoría de información financiera. Bogotá: Buena semilla.

Morales, A. (2013). Auditoría de Gestión. Monterrey: Cofae.

Muñoz, C. (2002). Auditoría en sistemas computacionales. México: Pearson.

Paredes, J. (2015). Auditoría I. Lima: Contabilidad para todos.

Plasencia, C. (2010). Sistema de control interno: garantía del logro de los objetivos. Scientific Electronic Library Online, Scielo, 1-3.

Sánchez, G. (2015). Auditoría de estados financieros. México: Pearson.

Tapia, C., Guevara, E., Castillo, S., Rojas, M., \& Salomón, L. (2016). Fundamentos de auditoría. México: Pearson.

Varela, E., Venini, A., \& Scarabino, J. (2013). Normas de auditoría y control interno. Red de revistas científicas de América Latina, El Caribe, España y Portugal, 90-93.

Venegas, W. (2007). El trabajo de campo en auditoría. San José: Universidad Estatal a Distancia, EUNED. Obtenido de https://books.google.com.ec/books?id=d5cMARyrSBMC\&pg=PA139\&dq=progra 
mas+de+trabajo+en+auditoria\&hl=es-

419\&sa=X\&ved=0ahUKEwj5j7qG59TiAhVHs1kKHdkgBjIQ6AEILjAB\#v=onepa ge \&q=programas $\% 20 \mathrm{de} \% 20$ trabajo $\% 20 \mathrm{en} \% 20$ auditoria $\& \mathrm{f}=$ false 


\section{PARA CITAR EL ARTÍCULO INDEXADO.}

Benavides Ortega, R., Narváez Zurita, C., Erazo Álvarez, J., \& Ordoñez Parra, Y. (2019). Auditoría financiera como herramienta de análisis de los estados financieros de la empresa Imgrumasa S.A. de la ciudad de Machala, periodo 2018. Visionario Digital, 3(2.1.), 577-598. https://doi.org/10.33262/visionariodigital.v3i2.1.589

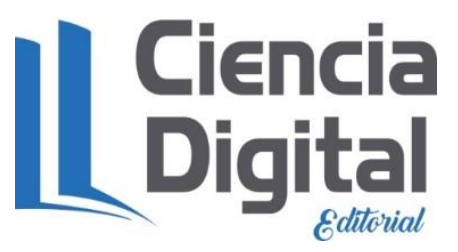

El artículo que se publica es de exclusiva responsabilidad de los autores y no necesariamente reflejan el pensamiento de la Revista Ciencia Digital.

El artículo queda en propiedad de la revista y, por tanto, su publicación parcial y/o total en otro medio tiene que ser autorizado por el director de la Revista Ciencia Digital.
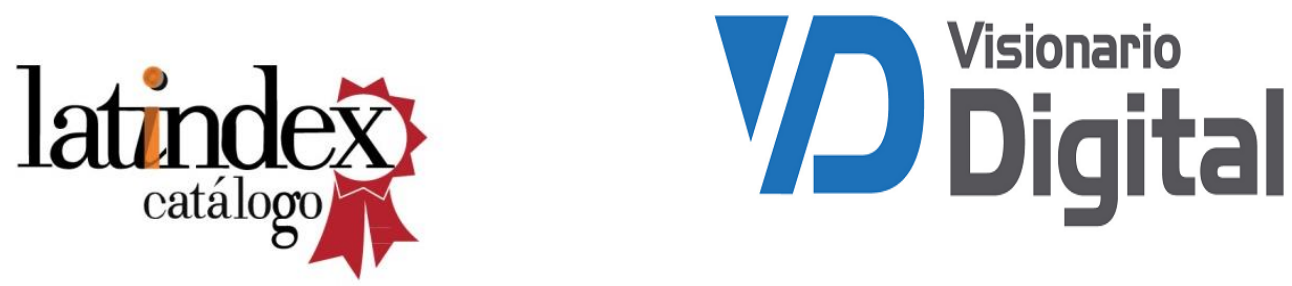